Article

\title{
Reassignment of the Structures of Products Produced by Reactions of the Product Believed To Be 2-(1-Phenyl-2- Thiocyanatoethylidene)-malononitrile with Electrophiles
}

\author{
Saleh Mohammed Al-Mousawi *, Moustafa Sherief Moustafa and Mohamed Hilmy Elnagdi \\ Department of Chemistry, Faculty of Science, University of Kuwait, Safat, 13060, P.O. Box 12613, \\ Kuwait
}

* Author to whom correspondence should be addressed; E-Mail: salehalmousawi@hotmail.com; Tel.: +965-24987081; Fax: +965-24816482.

Received: 1 April 2011; in revised form: 15 April 2011 / Accepted: 18 April 2011 /

Published: 26 April 2011

\begin{abstract}
The reactivity of the product believed to be 2-(1-phenyl-2-thiocyanatoethylidene)malononitrile toward a variety of electrophilic and nucleophilic reagents is reported.
\end{abstract}

Keywords: 2-(1-phenyl-2-thiocyanatoethylidene)malononitrile; thiazole; diaminopyrazole; pyrazolo $[1,5-\alpha]$ pyrimidinediamine

\section{Introduction}

Conflicting results have been reported for the structures of the products formed upon reaction of 2thiocyanatoethanones (1) with active methylene nitriles. In 1986, Abdelrazek et al [1] proposed that reaction of 1a with malononitrile in refluxing ethanol in the presence of catalytic piperidine affords the dicyanomethylidene adduct 2a. Recently a patent described the formation of the thiazole 3a from these substrates under very similar conditions (Scheme 1) [2]. Abdelrazek subsequently [3] reported that 2a could also be produced by reaction of the brominated dicyanomethylidene $4 \mathbf{a}$ with potassium thiocyanate, although a detailed procedure for this transformation was not provided. This observation served as evidence against the formation of $3 \mathbf{a}$ as the product of the reaction of $1 \mathbf{a}$ with malononitrile. Thiazole 3a was also reported to form in the reaction between 1a and malononitrile in presence of $\mathrm{KOH}[2,4]$. 
Scheme 1. Syntheses of compounds 3a,b and 7.

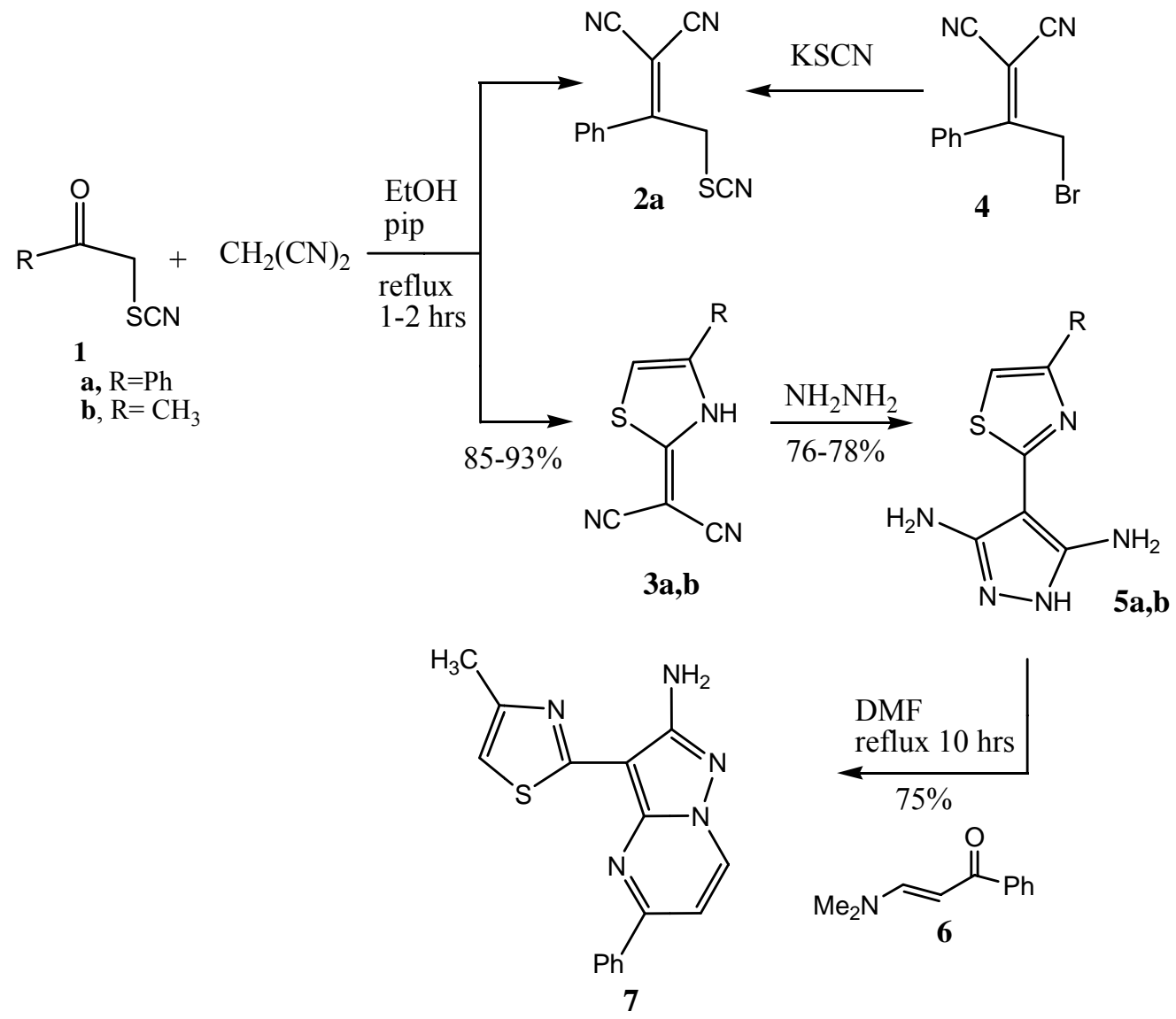

\section{Results and Discussion}

Scheme 1

We recently [5] confirmed that in fact the product generated in the reaction of $\mathbf{1 b}$ with malononitrile is $\mathbf{3 b}$ by X-ray crystallographic analysis of the thiazolylpyrazolo[1,5-a]pyrimidine derivative 7 [6], which was produced via reaction of $\mathbf{3 b}$ with hydrazine hydrate to yield the diaminopyrazole $\mathbf{5 b}$ and subsequent reaction of $\mathbf{5 b}$ with enaminone $\mathbf{6}$ (Figure 1 ).

Figure 1. Plot of the X-ray crystallographic data of pyrazolo[1,a]pyrimidine 7.

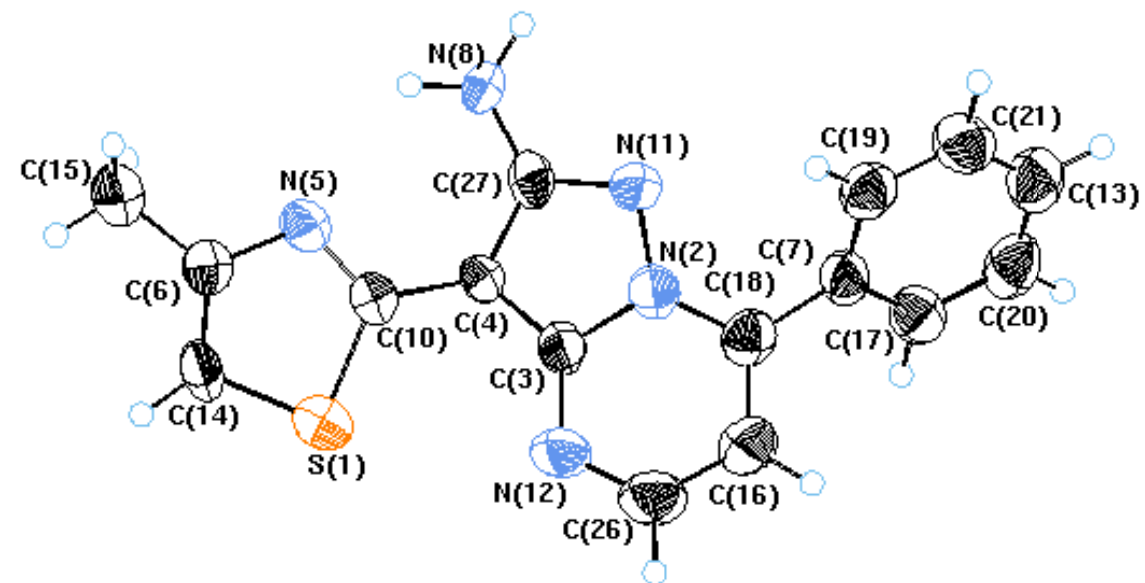


Although the diaminopyrazole 5a, produced by Salah Eldine [4] through the reaction of hydrazine hydrate with 3a, proved to be identical with the substance we produced by reaction of 1a with malononitrile, it was claimed that both $\mathbf{2}$ and $\mathbf{3}$ can be formed in this process. Consequently, we thought that a further effort aimed at confirming the structures of $\mathbf{5 a}, \mathbf{b}$ was in order since Abdelrazek has reported that 2a can be utilized as a precursor in syntheses of several heterocyclic substances. Thus, if $\mathbf{2 a}$ is really $\mathbf{3 a}$ the structures of products claimed to be formed from $\mathbf{2 a}$ in the earlier investigations need to be reassigned [1,3,7-9].

In the current work, we explored the reaction of diaminopyrazole 5a with benzylidenemalononitrile 8. This process generates a product, resulting from sequential addition and molecular hydrogen elimination, that could be assigned as either $\mathbf{1 1}$ or $\mathbf{1 4}$. These substances would be produced via the respective initially formed adducts 9 or 12 (Scheme 2).

Scheme 2. Syntheis of compound 11.
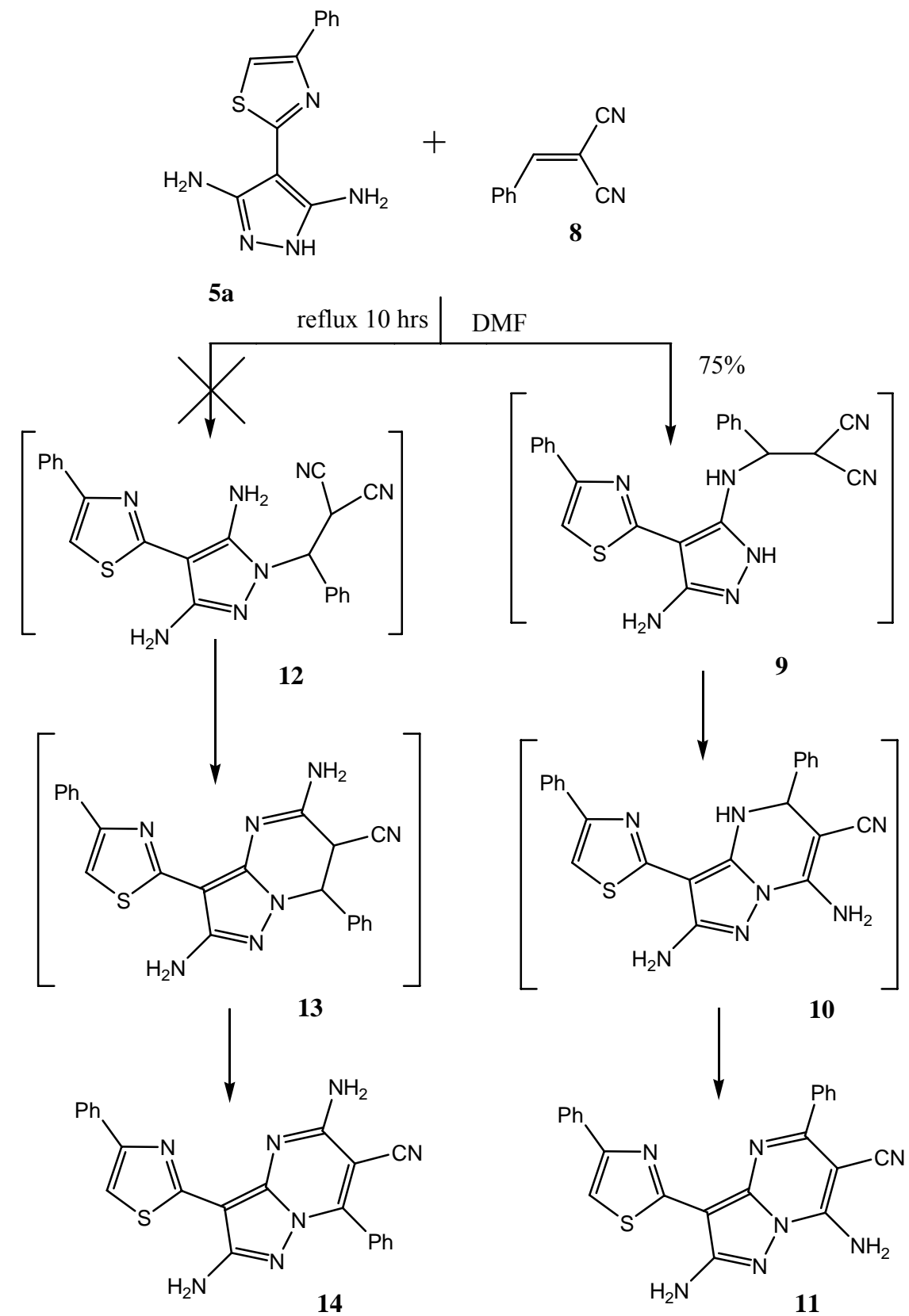
Cyclization of these intermediates would form 10 or 13, which then aromatize to generate 11 or 14, respectively The actual structure of the final product was shown to be compound 11, based on an analysis of ${ }^{15} \mathrm{~N}$ HMBC results. These results showed that the amino nitrogen at $\delta=30 \mathrm{ppm}$ is coupled to the bridgehead nitrogen at $\delta=211 \mathrm{ppm}$.

Diaminopyrazole 5a reacts with enaminonitrile 15 to yield the pyrazolo[1,5-a] pyrimidinediamine 17 (Scheme 3). The isomeric adduct 16 was also excluded as the product based on the results of ${ }^{15} \mathrm{~N}$ HMBC and the X-ray crystallographic analyses (Figure 2) [10].

Scheme 3. Syntheis of compound 17.
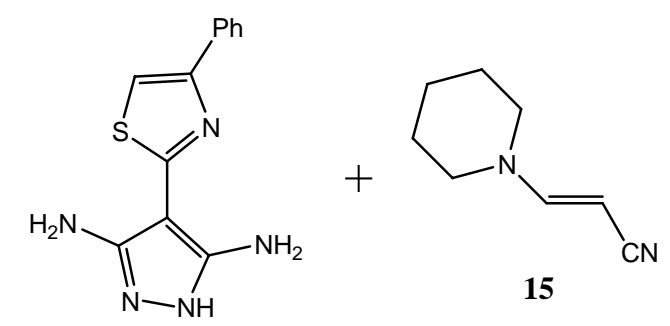

15

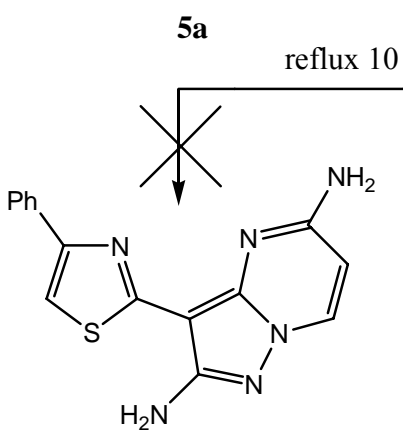

16

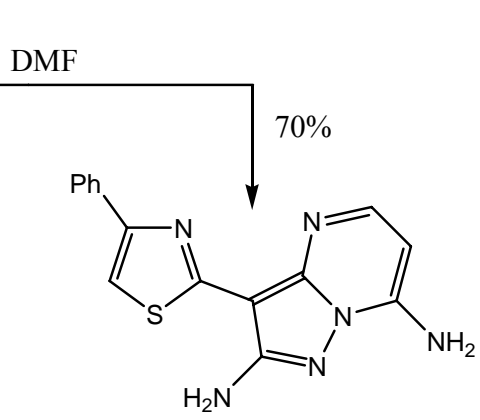

17

Figure 2. Plot of the x-ray crystallographic data of 3-(4-phenylthiazol-2-yl)pyrazolo[1,5a]pyrimidine-2,7-diamine (17).

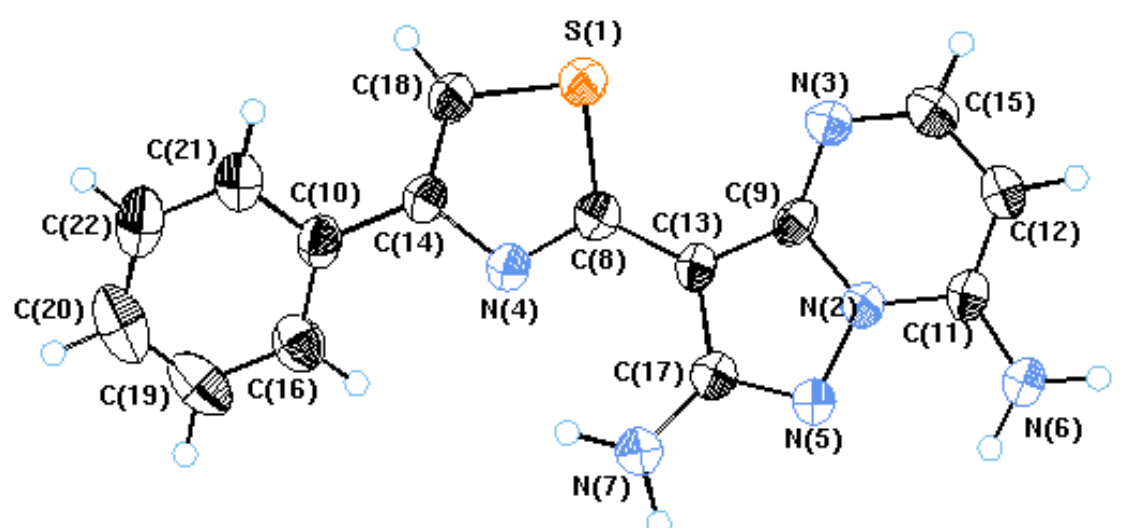

The combined observations made in this investigation confirm the structural assignment of 3a. Moreover, thiazole 3a undergoes a coupling reaction with benzenediazonium chloride to yield the aryldiazo derivative 18. Compound 18 was reduced by treatment with $\mathrm{Zn}$ metal in acetic acid to yield the amide 19 rather than the isomeric substance 20. This assignment is based on the absence of amine 
signals in both the IR and ${ }^{1} \mathrm{H}-\mathrm{NMR}$ spectra of 19. In addition, treatment of 3a with sulfuric acid in acetic acid led to production of the amide 21. Although 21 can exist in either a $E$ - or $Z$-isomeric form, the $E$-stereoisomer appears to be generated selectively, as indicated from NOE difference experiments thus irradiating $\mathrm{NH}$ at $\delta=12.46 \mathrm{ppm}$ has enhanced amide $\mathrm{NH}_{2}$ at $\delta=6.64 \mathrm{ppm}$ and vice versa, such enhancement cannot occur with Z-isomeric. Clearly the claim [3] that pyrrole 23 and thiophene 24 are produced from a substance assumed to be 2 must be subjected to more concrete verification (Scheme 4).

Scheme 4. Syntheses of compound 19 and 21.
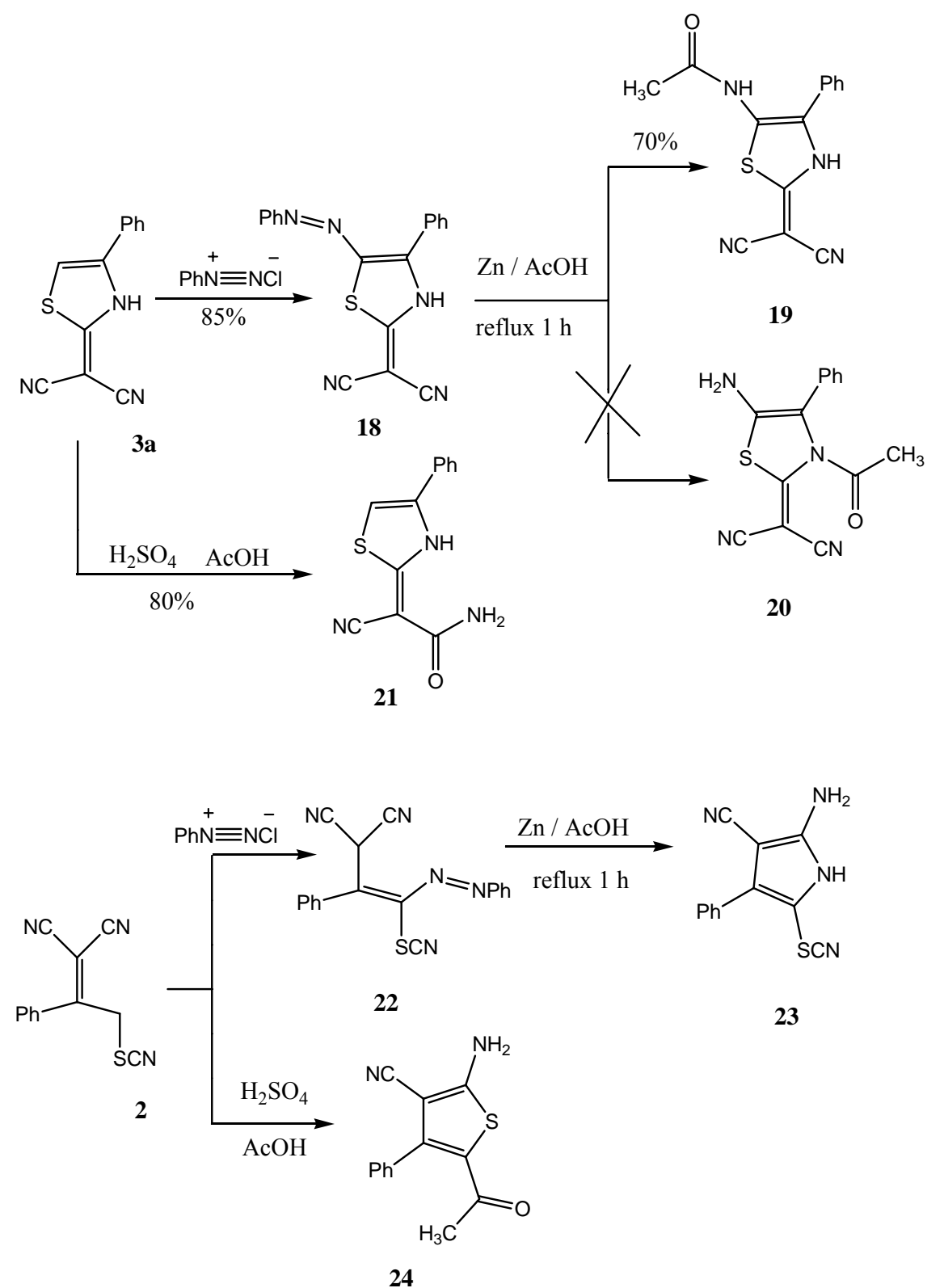

Finally, in an attempt to extend the Salah Eldine [4] reaction of 3a with furfurylidine malononitrile to its reaction with benzylidenemalononitrile $\mathbf{8}$, only $\mathbf{2 7}$ was generated via the intermediacy of adduct 26. The elimination of active methylene carbanions from substances analogous to $\mathbf{2 6}$ is well known [11] (Scheme 5). 
Scheme 5. Synthesis of compound 27.<smiles>N#CC(C#N)=C1NC(c2ccccc2)=CS1</smiles>

3a<smiles>N#CC1=C(N)C(c2ccccc2)C(c2ccccc2)N2C=CSC1=C2</smiles>

$\mathrm{EtOH} /$ pip reflux 3 hrs

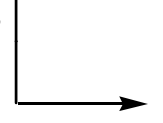<smiles>N#CC(C#N)=C1NC(=C2CCCCC2)C(C#N)C1c1ccccc1</smiles>

26

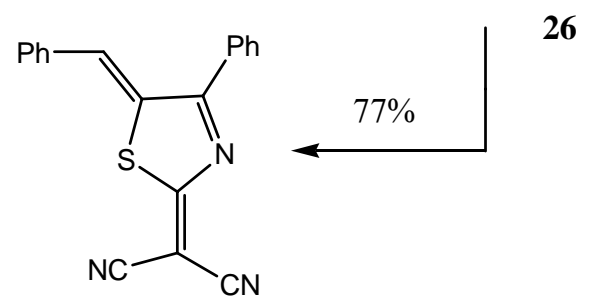

27

Abdelrazek has also reported [12] that 1-phenyl-2-thiocyanatoethanone (1a) reacts with ethyl cyanoacetate to yield either the alkylidene or the thiazole adducts 29 or 30, respectively. Again, analysis of the reaction mixture formed by carrying out this process under a variety of conditions revealed that $\mathbf{2 9}$ was not formed and only $\mathbf{3 0}$ was produced when potassium hydroxide was present (Scheme 6).

Scheme 6. Synthesis of compound 30.

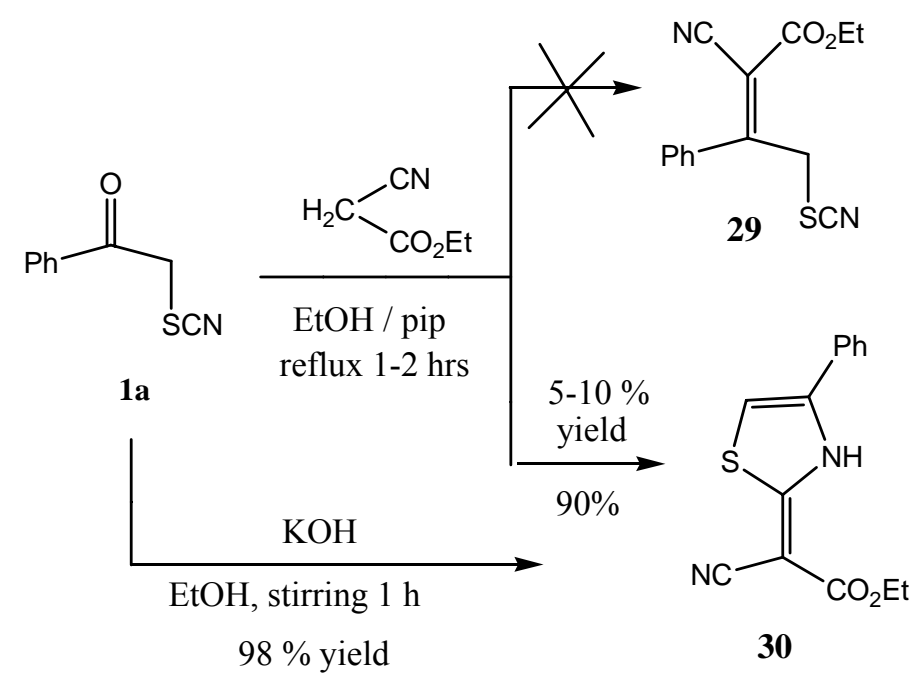


Our attention next shifted to an exploration of condensation reactions of $\mathbf{1 a}$ with other active methylene reagents. However, in our hands 1a failed to produce adducts with acetylacetone, ethyl acetoacetate and the malononitrile dimer. under a variety of conditions reactions. Only the self tricondensation of $\mathbf{3 1}$ ocurred in the case of reaction of cyanoketone $\mathbf{3 1}$ with 1a. Although the tricondensation product is well known, Briel et al. [13] recently assigned structure 32 to this substance. In contrast, Elnagdi et al. [14] have assigned the structure of this product as 33, a substance which in fact can also be formed by cyclization of 32. Again, Abdelrazek [15] has incorrectly claimed that this product is 33. Of course, MS analysis shows that 33 differs from both 32 and $\mathbf{3 4}$ (Scheme 7). It is possible that Briel's structural assignement is correct since heating the tricondensation product with zeolites affords an isomeric product that has been assigned as $\mathbf{3 3}$.

Scheme 7. Synthesis of compound 33.

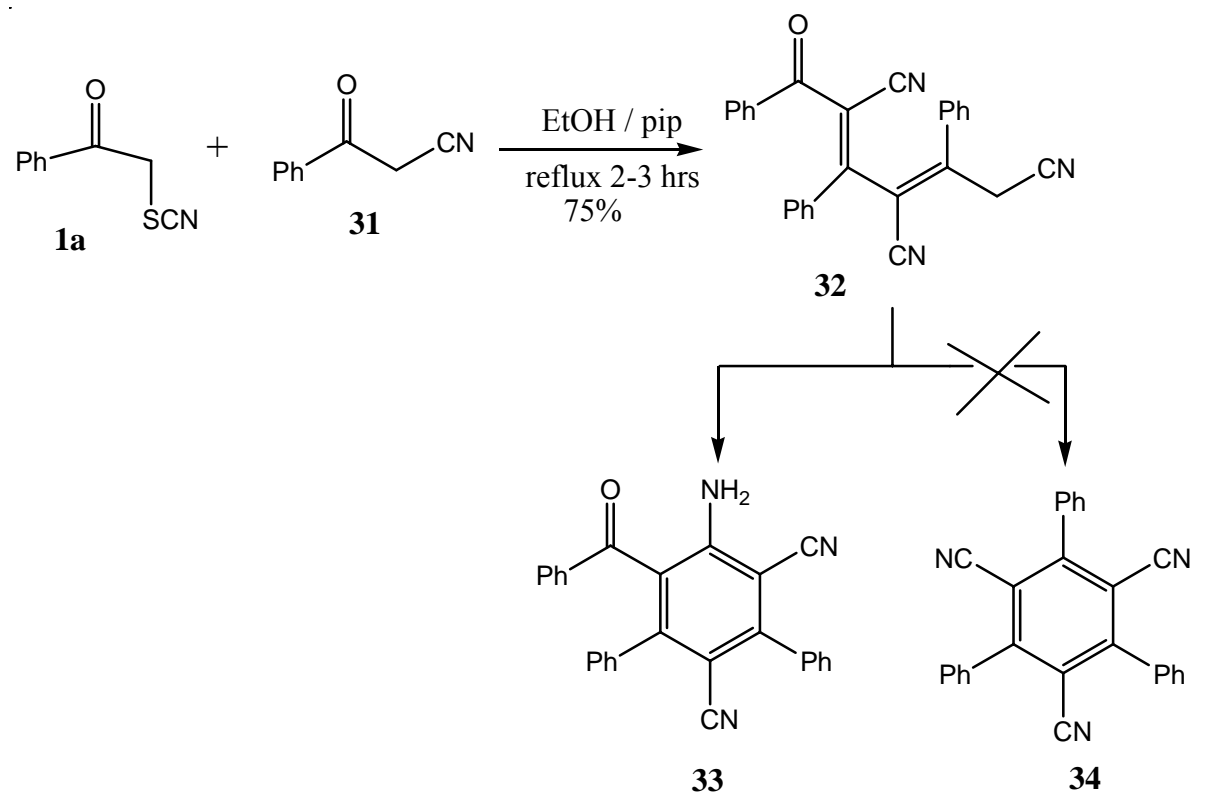

We have also investigated the reactivity of 1a with phenylhydrazine. This reaction has been reported by Abdelrazek et al. [8] to yield the hydrazone 35. In our hands, only the thiazole 36 was isolated from the reaction mixture (Scheme 8).

Scheme 8. Synthesis of compound 36 .

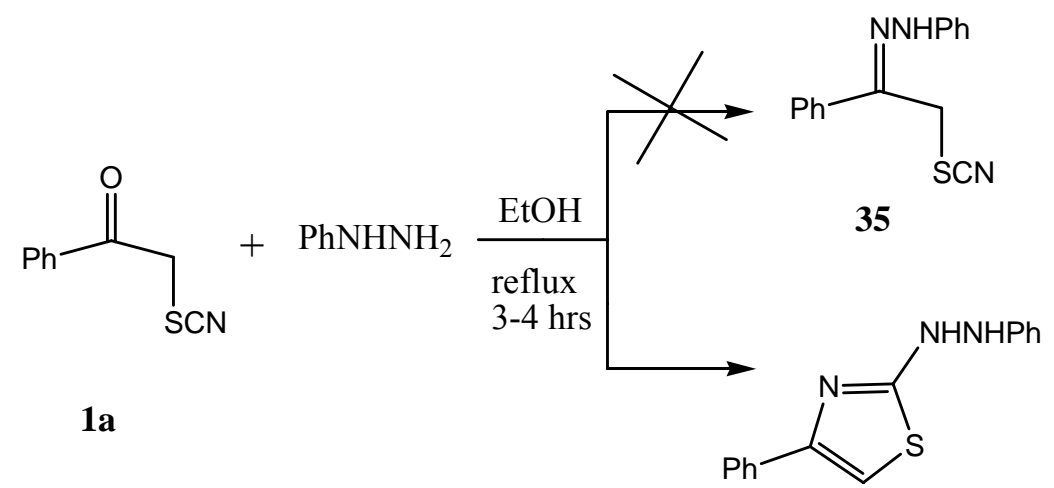




\section{Experimental}

\subsection{General}

Melting points are reported uncorrected and were determined with a Sanyo (Gallaenkamp) instrument. Infrared spectra were recorded using $\mathrm{KBr}$ pellets and a Perkin-Elmer $2000 \mathrm{FT}-\mathrm{IR}$ instrument. ${ }^{1} \mathrm{H}$ - and ${ }^{13} \mathrm{C}$-NMR spectra were determined by using a Bruker DPX instrument at $400 \mathrm{MHz}$ for ${ }^{1} \mathrm{H}-\mathrm{NMR}$ and $100 \mathrm{MHz}$ for ${ }^{13} \mathrm{C}-\mathrm{NMR}$ and either $\mathrm{CDCl}_{3}$ or DMSO- $d_{6}$ solutions with TMS as internal standards. Chemical shifts are reported in $\delta(\mathrm{ppm})$. Mass spectra were measured using VG Autospec Q MS 30 and MS 9 (AEI) spectrometer, with the EI (70 EV) mode. Elemental analyses were carried out by using a LEOCHNS-932 Elemental Analyzer.

\subsection{General Procedure for the Syntheses of 3a,b}

Solutions of malononitrile $(0.66 \mathrm{~g}, 0.01 \mathrm{~mol})$ and $\alpha$-thiocyanatoketones 1a,b $(0.01 \mathrm{~mol})$ in ethanol $(15 \mathrm{~mL}$ ) containing piperidine (5 drops) were stirred at reflux for 1-2 h. (completion assessed by TLC, 1:1 ethyl acetate-petroleum ether). The solid products, isolated by pouring the reaction mixtures into ice-water and subsequent separation by filtration, were crystallized from $\mathrm{EtOH}$ to afford green crystals.

2-(4-Phenylthiazol-2(3H)-ylidene)malononitrile (3a). Yield 93\%; m.p. 275-276 ${ }^{\circ} \mathrm{C}$; Anal. calcd. for $\mathrm{C}_{12} \mathrm{H}_{7} \mathrm{~N}_{3} \mathrm{~S}$ (225.27): C, 63.98; H, 3.13; N, 18.65; S, 14.23. Found: C, 63.94; H, 3.31; N; 18.45; S, 13.92; IR (KBr): $v_{\max } 3147(\mathrm{NH}), 2210(\mathrm{CN}), 2175(\mathrm{CN}) ;{ }^{1} \mathrm{H}-\mathrm{NMR}$ (DMSO): $\delta$, ppm 7.33 (s, 1H, CH), 7.45-7.49 (m, 3H, Ar-H), 7.71-7.72 (m, 2H, Ar-H), 13.23 (br, 1H, NH, $\mathrm{D}_{2} \mathrm{O}$ exchangeable); ${ }^{13} \mathrm{C}-\mathrm{NMR}$ (DMSO): $\delta$, ppm 172.27, 143.70, 130.01, 129.12(2C), 128.92, 127.51 (2C), 127.64, 117.75, 105.93 (2CN). MS: m/z (\%) 225 (M+100), 180 (20), 134 (45), 108 (10), 102 (15), 89 (15), 77 (10).

2-(4-Methylthiazol-2(3H)-ylidene)malononitrile (3b). Yield 85\%; m.p. 290-291 ${ }^{\circ} \mathrm{C}$; Anal. calcd. for $\mathrm{C}_{7} \mathrm{H}_{5} \mathrm{~N}_{3} \mathrm{~S}$ (163.2): C, 51.52; H, 3.09; N, 25.75; S, 19.64. Found: C, 51.26; H, 3.12; N; 25.54; S, 19.27; IR (KBr): $v_{\max } 3160(\mathrm{NH}), 2179(2 \mathrm{CN}) ;{ }^{1} \mathrm{H}-\mathrm{NMR}$ (DMSO): $\delta$, ppm $2.17\left(\mathrm{~s}, 3 \mathrm{H}, \mathrm{CH}_{3}\right), 6.71(\mathrm{~s}, 1 \mathrm{H}$, $\mathrm{CH}$ ), 13.1 (br, 1H, NH, D $\mathrm{D}_{2}$ exchangeable); ${ }^{13} \mathrm{C}-\mathrm{NMR}$ (DMSO): $\delta$, ppm 170.10, 142.54, 105.93 $(2 \mathrm{CN}), 95.23,87.68,16.54 . \mathrm{MS}: \mathrm{m} / \mathrm{z}(\%) 163\left(\mathrm{M}^{+}, 100\right), 136(20), 118(30), 98(10), 71(50)$.

\subsection{General Procedure for the Syntheses of $\mathbf{5 a , b}$}

Mixtures of 3a,b $(0.01 \mathrm{~mol})$ and hydrazine monohydrate $(0.50 \mathrm{~g}, 0.01 \mathrm{~mol})$ in DMF $(10 \mathrm{~mL})$ were stirred at reflux for $20 \mathrm{~h}$. (completion assessed by TLC analysis using ethyl acetate-petroleum ether 1:1). The mixtures were cooled and poured into ice-water. The solid products, collected by filtration, were crystallized from DMF to give light yellow crystals.

4-(4-Phenylthiazol-2-yl)-1H-pyrazole-3,5-diamine (5a). Yield 78\%; m.p. 322-323 ${ }^{\circ} \mathrm{C}$; Anal. calcd. for $\mathrm{C}_{12} \mathrm{H}_{11} \mathrm{~N}_{5} \mathrm{~S}$ (257.31): C, 56.01; H, 4.31; N, 27.22; S, 12.41. Found: C, 55.80; H, 4.41; N; 26.88; S, 11.99; IR (KBr): $v_{\max } 3372,3256\left(\mathrm{NH}_{2}\right), 3176,3112\left(\mathrm{NH}_{2}\right), 3132(\mathrm{NH}) ;{ }^{1} \mathrm{H}-\mathrm{NMR}$ (DMSO): $\delta$, ppm 5.39 (br, 4H, 2NH, $\mathrm{D}_{2} \mathrm{O}$ exchangeable), 7.31-7.46 (m, 3H, Ar-H), $7.75(\mathrm{~s}, 1 \mathrm{H}, \mathrm{CH}), 7.96-7.98$ (m, 
2H, Ar-H), 10.73 (br, 1H, NH, $\mathrm{D}_{2} \mathrm{O}$ exchangeable); ${ }^{13} \mathrm{C}-\mathrm{NMR}$ (DMSO): $\delta$, ppm 166.29, 162.08, 152.30 (2C), 134.31, 128.69 (2C), 127.69, 125.89 (2C), 107.48, 87.97. MS: m/z (\%) $257\left(\mathrm{M}^{+}, 100\right), 226$ (10), 200 (5), 134 (35), 128 (10), 90 (10).

4-(4-Methylthiazol-2-yl)-1H-pyrazole-3,5-diamine (5b). Yield 76\%; m.p. 330-332 ${ }^{\circ} \mathrm{C}$; Anal. calcd. for $\mathrm{C}_{7} \mathrm{H}_{9} \mathrm{~N}_{5} \mathrm{~S}$ (195.24): C, 43.06; H, 4.65; N, 35.87; S, 16.42. Found: C, 42.89; H, 4.73; N; 35.60; S, 15.98; IR (KBr): $U_{\max }$ 3371, $3275\left(\mathrm{NH}_{2}\right), 3255,3180\left(\mathrm{NH}_{2}\right), 3118(\mathrm{NH}) ;{ }^{1} \mathrm{H}-\mathrm{NMR}$ (DMSO): $\delta$, ppm 2.23 (s, $\left.3 \mathrm{H}, \mathrm{CH}_{3}\right), 5.62\left(\mathrm{br}, 4 \mathrm{H}, 2 \mathrm{NH}_{2}, \mathrm{D}_{2} \mathrm{O}\right.$ exchangeable), $6.86(\mathrm{~s}, 1 \mathrm{H}, \mathrm{CH}), 10.67$ (br, $1 \mathrm{H}, \mathrm{NH}, \mathrm{D}_{2} \mathrm{O}$ exchangeable); ${ }^{13} \mathrm{C}-\mathrm{NMR}$ (DMSO): $\delta$, ppm 164.33, 161.56, 150.03 (2C), 106.88, 87.68, 16.84. MS: $\mathrm{m} / \mathrm{z}(\%) 195\left(\mathrm{M}^{+}, 100\right), 164(20), 138(10), 123$ (15), 112 (5), 72 (15).

\subsection{Synthesis of 2,7-diamino-5-phenyl-3-(4-phenylthiazol-2-yl)pyrazolo[1,5-a]pyrimidine-6- carbonitrile (11)}

A mixture of 5a $(2.57 \mathrm{~g}, 0.01 \mathrm{~mol})$ and benzylidenemalononitrile $(\mathbf{8}, 1.54 \mathrm{~g}, 0.01 \mathrm{~mol})$ in DMF $(10 \mathrm{~mL}$ ) was stirred at reflux for $10 \mathrm{~h}$ (completion assessed by TLC analysis using ethyl acetatepetroleum ether 1:1 as eluent). The reaction mixture was cooled and poured into ice-water giving a solid which was collected by filtration and crystallized from DMF to give a the product as yellow crystals in a yield of $75 \%$; m.p. $298-300{ }^{\circ} \mathrm{C}$; Anal. calcd. for $\mathrm{C}_{22} \mathrm{H}_{15} \mathrm{~N}_{7} \mathrm{~S}$ (409.47): C, 64.53; H, 3.69; N, 23.94; S, 7.83. Found: C, 64.61; H, 3.69; N; 23.79; S, 7.59; IR (KBr): $v_{\max } 3430,3318\left(\mathrm{NH}_{2}\right), 3429$, $3315\left(\mathrm{NH}_{2}\right), 2211(\mathrm{CN}) ;{ }^{1} \mathrm{H}-\mathrm{NMR}$ (DMSO): $\delta$, ppm 6.85 (br, 2H, $\mathrm{NH}_{2}, \mathrm{D}_{2} \mathrm{O}$ exchangeable), 7.35-8.03 $\left(\mathrm{m}, 11 \mathrm{H}, \mathrm{Ar}-\mathrm{H}\right.$, thiazole-H), 8.57 (br, $2 \mathrm{H}, \mathrm{NH}_{2}, \mathrm{D}_{2} \mathrm{O}$ exchangeable); ${ }^{13} \mathrm{C}-\mathrm{NMR}$ (DMSO): $\delta, \mathrm{ppm}$ $195.47,159.46,157.98,152.59,148.91,145.21,137.10,133.97,130.37$ (2C), 128.77 (2C), 128.70 (2C), 128.38 (2C), 127.89, 125.93, 116.48, 111.39, 92.24, 73.15. MS: m/z (\%) $409\left(\mathrm{M}^{+}, 100\right), 333$ (5), 204 (15), 195 (5), $134(30), 90(5)$.

\subsection{Synthesis of 3-(4-phenylthiazol-2-yl)pyrazolo[1,5-a]pyrimidine-2,7-diamine (17)}

A mixture of 5a $(2.57 \mathrm{~g}, 0.01 \mathrm{~mol})$ and 3-(piperidin-1-yl)acrylonitrile (15, $1.36 \mathrm{~g}, 0.01 \mathrm{~mol})$ in DMF $(10 \mathrm{~mL}$ ) was stirred at reflux for $10 \mathrm{~h}$ (completion assessed by TLC analysis using ethyl acetatepetroleum ether 1:1 as eluent). The reaction mixture was cooled and poured into ice-water giving a solid which was collected by filtration and crystallized from DMF to give a the product as yellow crystals in a yield of $70 \%$; m.p. $329-330{ }^{\circ} \mathrm{C}$; Anal. calcd. for $\mathrm{C}_{15} \mathrm{H}_{12} \mathrm{~N}_{6} \mathrm{~S}$ (308.36): C, 58.43; H, 3.92; N, 27.25; S, 10.40. Found: C, 58.33; H, 3.79; N; 27.20; S, 10.37; IR (KBr): $v_{\max } 3443,3305\left(\mathrm{NH}_{2}\right)$, 3355, $3263\left(\mathrm{NH}_{2}\right) ;{ }^{1} \mathrm{H}-\mathrm{NMR}$ (DMSO): $\delta$, ppm 6.13 (d, $\left.1 \mathrm{H}, J=6 \mathrm{~Hz}, \mathrm{CH}\right), 6.62\left(\mathrm{br}, 2 \mathrm{H}, \mathrm{NH}_{2}, \mathrm{D}_{2} \mathrm{O}\right.$ exchangeable), 7.33-7.47 (m, 3H, Ar-Hs), 7.61 (br, 2H, $\mathrm{NH}_{2}, \mathrm{D}_{2} \mathrm{O}$ exchangeable), $7.85(\mathrm{~s}, 1 \mathrm{H}$, thiazole-H), $8.01(\mathrm{~m}, 2 \mathrm{H}, \mathrm{Ar}-\mathrm{Hs}), 8.07$ (d, 1H, $J=6 \mathrm{~Hz}, \mathrm{CH}) ;{ }^{13} \mathrm{C}-\mathrm{NMR}$ (DMSO): $\delta$, ppm 160.59, 156.98, 152.22, 149.36, 147.14, 146.94, 134.31, 128.75 (2C), 127.72, 125.90 (2C), 109.66, 89.99, 88.59. MS: m/z (\%) $308\left(\mathrm{M}^{+}, 100\right), 268$ (30), 175 (5), 154 (5), 134 (30), 102 (5), 89(5), 77 (5). 


\subsection{Synthesis of 2-(4-Phenyl-5-phenylazo-3H-thiazol-2-ylidene)malononitrile (18a)}

A cold solution of benzenediazonium chloride $(0.01 \mathrm{~mol})$ was prepared by adding a solution of sodium nitrite $\left(0.7 \mathrm{~g}\right.$ in $\left.10 \mathrm{~mL} \mathrm{H}_{2} \mathrm{O}\right)$ to a cold solution of aniline hydrochloride $(0.93 \mathrm{~g}, 0.01 \mathrm{~mol}$ of aniline in $5 \mathrm{~mL}$ concentrated $\mathrm{HC} 1$ ) with stirring at room temperature. The resulting solution was then added to cold solutions of 3a $(2.25 \mathrm{~g}, 0.01 \mathrm{~mol})$ in ethanol $(50 \mathrm{~mL})$ containing sodium acetate $(2 \mathrm{~g})$. The reaction mixtures was stirred for $1 \mathrm{~h}$ and then filtered. The solid products were crystallized from EtOH to give the products as red crystals, yield 85\%; m.p. 198-200 ${ }^{\circ} \mathrm{C}$; Anal. calcd. for $\mathrm{C}_{18} \mathrm{H}_{11} \mathrm{~N}_{5} \mathrm{~S}$ (329.38): C, 65.64; H, 3.37; N, 21.26; S, 9.73. Found: C, 65.49; H, 3.51; N; 21.15; S, 9.39; IR (KBr): $\mathrm{U}_{\max } 3180(\mathrm{NH}), 2216(2 \mathrm{CN}) ;{ }^{1} \mathrm{H}-\mathrm{NMR}$ (DMSO): $\delta$, ppm 5.03 (br, 1H, NH, $\mathrm{D}_{2} \mathrm{O}$ exchangeable), 7.25-7.60 (m, 8H, Ar-H), 8.22-8.24 (m, 2H, Ar-H); ${ }^{13} \mathrm{C}-\mathrm{NMR}$ (DMSO): $\delta$, ppm 175.46, 147.29, 138.67, 131.79, 131.47, 130.73 (2C), 130.70 (2C), 129.59 (2C), 128.63 (2C), 127.05, 118.96, 117.71, 115.70 (2CN). MS: m/z (\%) 329 (M+, 100), 301 (20), 237 (15), 225 (25), 153 (5), 103 (20), 92 (25), 77 (65).

\subsection{Synthesis of N-(2-Dicyanomethylene-4-phenyl-2,3-dihydro-thiazol-5-yl)-acetamide (19)}

A mixture of 18a $(3.29 \mathrm{~g}, 0.01 \mathrm{~mol})$ and $\mathrm{Zn}$ dust $(1 \mathrm{~g})$ in $\mathrm{AcOH}(10 \mathrm{~mL})$ was stirred at reflux for 1 hr (completion assessed by TLC analysis using ethyl acetate-petroleum ether 1:1 as eluent). The reaction mixture was cooled and poured into ice-water giving a solid which was collected by filtration and crystallized from EtOH to give the product as faint green coloured crystals in a yield of 70\%; m.p. 335-338 ${ }^{\circ} \mathrm{C}$; Anal. calcd. for $\mathrm{C}_{14} \mathrm{H}_{10} \mathrm{~N}_{4} \mathrm{OS}$ (282.32): C, 59.56; H, 3.57; N, 19.85; S, 11.36. Found: C, 59.61; H, 3.44; N; 19.75; S, 11.42; IR (KBr): $v_{\max } 3057(\mathrm{NH}), 3027(\mathrm{NH}), 2204(\mathrm{CN}), 2178(\mathrm{CN}) ;{ }^{1} \mathrm{H}-$ NMR (DMSO): $\delta$, ppm 2.02 (s, 3H, $\left.\mathrm{CH}_{3}\right), 7.21-7.76$ (m, 6H, Ar-Hs, NH, $\mathrm{D}_{2} \mathrm{O}$ exchangeable), 10.07 (br, 1H, NH, $\mathrm{D}_{2} \mathrm{O}$ exchangeable); ${ }^{13} \mathrm{C}-\mathrm{NMR}$ (DMSO): $\delta$, ppm 169.86, 169.04, 164.93, 163.14, 128.69, 128.28 (2C), 128.09, 127.89 (2C), 127.73, 119.92, 117.67, 22.39. MS: m/z (\%) $282\left(\mathrm{M}^{+}, 60\right), 257$ (20), 240 (100), 215 (85), 180 (10), 148(30), 121 (40), 104 (75), 93 (40), 77 (65), 73 (40).

\subsection{Synthesis of 2-cyano-2-(4-phenylthiazol-2(3H)-ylidene)acetamide (21)}

A mixture of 3a $(2.25 \mathrm{~g}, 0.01 \mathrm{~mol})$ and $\mathrm{H}_{2} \mathrm{SO}_{4}(3 \mathrm{~mL})$ in $\mathrm{AcOH}(10 \mathrm{~mL})$ was stirred at reflux for $1 \mathrm{~h}$. The reaction mixture was cooled and poured into ice-water giving a solid which was collected by filtration and crystallized from $\mathrm{AcOH}$ to give the product as white coloured crystals a yield of $80 \%$; m.p. 229-231 ${ }^{\circ} \mathrm{C}$; Anal. calcd. for $\mathrm{C}_{12} \mathrm{H}_{9} \mathrm{~N}_{3} \mathrm{OS}$ (243.28): C, 59.24; H, 3.73; N, 17.27; S, 13.18. Found: C, 59.15; H, 3.58; N; 17.34; S, 13.32; IR (KBr): $\mathrm{U}_{\max }$ 3305, $3236\left(\mathrm{NH}_{2}\right), 3126(\mathrm{NH}), 2184(\mathrm{CN}) ;{ }^{1} \mathrm{H}-$ NMR (DMSO): $\delta$, ppm 6.64 (br, 2H, NH, $\mathrm{D}_{2} \mathrm{O}$ exchangeable), 7.15 (s, 1H, thiazole-H), 7.43-7.68 (m, $5 \mathrm{H}, \mathrm{Ar}-\mathrm{H}$ ), 12.46 (br, 1H, NH, $\mathrm{D}_{2} \mathrm{O}$ exchangeable); ${ }^{13} \mathrm{C}-\mathrm{NMR}$ (DMSO): $\delta$, ppm 169.77, 139.49, 129.37, 129.27, 128.93 (2C), 126.83 (2C), 126.07, 125.22, 118.94, 106.97. MS: m/z (\%) $243\left(\mathrm{M}^{+}, 90\right)$, 226 (100), 200 (45), 171 (5), 134 (40), 102 (45), 98 (10), 77 (5).

\subsection{Synthesis of 2-(5-benzylidene-4-phenylthiazol-2(5H)-ylidene)malononitrile (27)}

A mixture of 3a $(2.25 \mathrm{~g}, 0.01 \mathrm{~mol})$ and benzylidenemalononitrile $(\mathbf{8}, 1.54 \mathrm{~g}, 0.01 \mathrm{~mol})$ in $\mathrm{EtOH}$ $(20 \mathrm{~mL})$ in presence of piperidine $(1 \mathrm{~mL})$ was stirred at reflux for $3 \mathrm{~h}$. The reaction mixture was cooled and poured into ice-water giving a solid which was collected by filtration and crystallized from EtOH 
to give the product as faint yellow coloured crystals in a yield of $77 \%$; m.p. $280-282{ }^{\circ} \mathrm{C}$; Anal. calcd. for $\mathrm{C}_{19} \mathrm{H}_{11} \mathrm{~N}_{3} \mathrm{~S}$ (313.38): C, 72.82; H, 3.54; N, 13.41; S, 10.23. Found: C, 12.94; H, 3.32; N; 13.31; S, 10.19; IR (KBr): $v_{\max } 22.7(\mathrm{CN}), 2179(\mathrm{CN}) ;{ }^{1} \mathrm{H}-\mathrm{NMR}$ (DMSO): $\delta$, ppm 5.84 (s, 1H, CH), 7.15-7.42 (m, 10H, Ar-H); ${ }^{13} \mathrm{C}-\mathrm{NMR}$ (DMSO): $\delta$, ppm 169.07, 141.21, 140.04, 131.24, 130.57, 129.69, 129.21 (2C), 128.91 (2C), 128.45 (2C), 128.21, 127.94, 127.61 (2C), 124.62, 122.00, 117.17. MS: m/z (\%) $313\left(\mathrm{M}^{+}, 80\right), 285$ (10), 235 (90), 225 (100), $178(10), 134$ (60), $98(20)$.

\subsection{Synthesis of ethyl 2-cyano-2-(4-phenylthiazol-2(3H)-ylidene)acetate (30)}

Solutions of ethyl cyanoacetate $(1.13 \mathrm{~g}, 0.01 \mathrm{~mol})$ and $\alpha$-thiocyanatoketone $1 \mathrm{a}(0.01 \mathrm{~mol})$ in ethanol $(15 \mathrm{~mL})$ containing $\mathrm{KOH}(1 \mathrm{~g})$ were stirred at room temperature for $1-2 \mathrm{~h}$ (completion assessed by TLC, 1:1 ethyl acetate- petroleum ether). The solid products, produced by pouring the reaction mixtures into ice-water containing $\mathrm{HCl}(2 \mathrm{~mL})$ and subsequent separation by filtration, were crystallized from EtOH to give the product as white crystals in a yield of $90 \%$; m.p. $157-159{ }^{\circ} \mathrm{C}$; Anal. calcd. for $\mathrm{C}_{14} \mathrm{H}_{12} \mathrm{~N}_{2} \mathrm{O}_{2} \mathrm{~S}$ (272.32): C, 61.75; H, 4.44; N, 10.29; S, 11.77. Found: C, 61.71; H, 4.32; N; 10.18; S, 11.98; IR (KBr): $v_{\max } 3152(\mathrm{NH}), 2211(\mathrm{CN})$; ${ }^{1} \mathrm{H}-\mathrm{NMR}$ (DMSO): $\delta$, ppm 1.23 (t, $\left.3 \mathrm{H}, J=6 \mathrm{~Hz}, \mathrm{CH}_{3}\right), 4.16\left(\mathrm{q}, 2 \mathrm{H}, J=6 \mathrm{~Hz}, \mathrm{CH}_{2}\right), 7.15(\mathrm{~s}, 1 \mathrm{H}$, thiazole-H), 7.43-7.71 (m, 5H, Ar-H), 12.99 (br, $1 \mathrm{H}, \mathrm{NH}, \mathrm{D}_{2} \mathrm{O}$ exchangeable); ${ }^{13} \mathrm{C}-\mathrm{NMR}$ (DMSO): $\delta$, ppm 169.23, 166.55, 140.68, 129.31 (2C), 128.75, 128.75 (2C), 127.00, 117.54, 107.49, 62.93, 59.68, 14.55. MS: m/z (\%) $272\left(\mathrm{M}^{+}, 90\right)$, 226 (100), 200 (50), 172 (10), 153 (5), 134 (50), 102 (40), 89 (10), 77 (10).

\subsection{Synthesis of 1-phenyl-2-(4-phenylthiazol-2-yl)hydrazine (36)}

A mixture of 1a $(1.77 \mathrm{~g}, 0.01 \mathrm{~mol})$, phenyl hydrazine $(1.80 \mathrm{~g}, 0.02 \mathrm{~mol})$, in EtOH $(20 \mathrm{~mL})$ was refluxed for 3-4 h (completion assessed by TLC analysis using ethyl acetate-petroleum ether 1:1 as eluent). The reaction mixture was cooled and poured into ice-water giving a solid which was collected by filtration and crystallized from $\mathrm{EtOH}$ to give the product as white crystals in a yield of 75\%; m.p. 216-218 ${ }^{\circ} \mathrm{C}$; Anal. calcd. for $\mathrm{C}_{15} \mathrm{H}_{13} \mathrm{~N}_{3} \mathrm{~S}$ (267.35): C, 67.39; H, 4.90; N, 15.72; S, 11.99. Found: C, 67.17; H, 4.88; N; 15.58; S, 12.12; IR (KBr): $v_{\max } 3239$ (NH), 3089 (NH); ${ }^{1} \mathrm{H}-\mathrm{NMR}$ (DMSO): $\delta$, ppm 6.47-7.61 (m, 11H, Ar-H, thiazole-H), 9.08 (br, 1H, NH, $\mathrm{D}_{2} \mathrm{O}$ exchangeable), 12.60 (br, 1H, NH, $\mathrm{D}_{2} \mathrm{O}$ exchangeable); ${ }^{13} \mathrm{C}-\mathrm{NMR}$ (DMSO): $\delta$, ppm 163.35, 147.00, 130.91, 128.94 (2C), 128.54 (2C), 128.01, 127.91, 126.59 (2C), 119.59, 112.59 (2C), 111.19. MS: m/z (\%) $267\left(\mathrm{M}^{+}, 100\right), 234$ (5), 207 (5), 175 (15), 148 (5), 117 (70), 93 (50), 77 (10).

\section{Conclusions}

In conclusion, the results of this study confirm that the substance thought to be 2-(1-phenyl-2thiocyanatoethylidene)malononitrile (2) is not generated in the reaction of ketone 1a with malononitrile. The product produced in this process is in fact the 2-(thiazol-2(3H)-ylidene)malononitriles 3a,b. If 2-(1-phenyl-2-thiocyanatoethylidene)-malononitrile (2) was really produced in this reaction, then an exact experimental procedure that would enable a reasonably skilled chemist to repeat the process should be published. Finally, this effort has provided the structures of some products arising from reactions of 3a,b with a variety of electrophilic and nucleophilic reagents. 


\section{Acknowledgements}

The authors are grateful to Kuwait University Research Administration for the financial support of project SC1/10. Financial support of Mr. Moustafa Sherief by College of Graduate Studies is highly appreciated. Analytical facilities provided by SAF projects No. GS 01/05 \& GS 03/01 are greatly appreciated.

\section{References and Notes}

1. Abdelrazek, F.M.; Fadda, A.A. Nitriles in heterocyclic synthesis-A novel synthesis of polyfunctionally substituted pyrrole derivatives. Z. Naturforsch. 1986, 41B, 499-501.

2. Ishiwata, Y. Heterocyclic azo dye with improved fastness, high extinction coefficients and good hue. EP1975204 (A2), 1 October 2008.

3. Abdelrazek, F.M.; Ead, H.A. Heterocyclic synthesis with nitriles: A new approach to thiophene and thieno[2,3-d]pyrimidine derivatives. J. Prakt. Chem. 1988, 330, 585-589.

4. Salah El-Din, A.M. Fused thiazole from 2-(4-phenyl-3(h)-thiazol-2-ylidene) malononitrile: a novel synthesis of thiazolo[3,2-c]pyrimidine, thiazolo[3,2-a]pyridine, pyrazolo[3,4-d]-1,3thiazolino[2,3-f]pyrimidine and arylazo thiazolylidene derivatives. Sulfur Lett. 2003, 26, 35-41.

5. Al-Mousawi, S.M.; Moustafa, M.S.; Elnagdi, M.H. Polyfunctional heteroaromatics: A route to dicyanomethylene thiazoles based on the reaction of $\alpha$-thiocyanatoketones with malononitrile. Arkivoc 2010, ii, 224-232.

6. CCDC 771782 contains the supplementary crystallographic data for compound 7 . These data can be obtained free of charge from the Cambridge Crystallographic Data Centre via www.ccdc.cam.ac.uk

7. Abdelrazek, F.M.; Salah El-Din, A.M.; Elbazza, Z.E. Synthesis of novel thieno[2,3-d]pyrimidine, thieno[2,3-b]pyridine and thiazolo[3,2-a]thieno[2,3-d]pyrimidine derivatives and their effect on the production of mycotoxins. Arch. Pharm. 1992, 325, 301-305.

8. Abdelrazek, F.M.; Kandeel, Z.E. The reaction of $\omega$-thiocyanatoacetophenones with hydrazines. Phosphor. Sulfur Silicon 1991, 62, 101-104.

9. Abdelrazek, F.M. Heterocyclic synthesis with nitriles: A novel synthesis of some thiophene and thieno[2,3-d]pyrimidine derivatives. Z. Naturforsch 1989, 44, 488-492.

10. CCDC 795153 contains the supplementary crystallographic data for compound $\mathbf{1 7}$. These data can be obtained free of charge from the Cambridge Crystallographic Data Centre via www.ccdc.cam.ac.uk

11. Girgis, N.S.; Elgemeie, G.E.H.; Nawar, G.A.; Elnagdi, M.H. $\alpha, \beta$-Unsaturated nitriles in heterocyclic synthesis: The reaction of $\beta$-(2-furanyl)- and $\beta$-(2-thienyl)acrylonitrile with active methylene reagents. Lieb. Ann. Chem. 1983, 9, 1468-1475.

12. Abdelrazek, F.M.; Shams, H.Z.; Elnagdi, M.H. Nitriles in heterocyclic synthesis: synthesis of 2substituted 4-phenylthiazoles from phenacyl thiocyanate. J. Chem. Res. 1985, 8, 246-247.

13. Briel, D.; Rybak, A.; Kronbach, C.; Unverfrth, K. Substituted 2-Aminothiopen-derivatives: A potential new class of GluR6-Antagonists. Eur. J. Med. Chem. 2010, 45, 69-77. 
14. Al-Awadi, N.A.; Abdelkhalik, M.M.; Abdelhamid, I.A.; Elnagdi, M.H. Pyrolytic methods in organic synthesis: novel routes for the synthesis of 3-oxoalkanenitriles, 2-acyl anilines, and 2aroyl anilines. Synlett 2007, 19, 2979-2982.

15. Abdelrazek, F.M.; Michael, F.A. New data about the reaction of benzyolacetonitrile with malononitrile and its self-condensation. J. Heterocycl. Chem. 2006, 43, 7-10.

Sample Availability: Samples of the all compounds are available from the authors.

(C) 2011 by the authors; licensee MDPI, Basel, Switzerland. This article is an open access article distributed under the terms and conditions of the Creative Commons Attribution license (http://creativecommons.org/licenses/by/3.0/). 